\title{
O USO DA CONSTRUÇÃO BITRANSITIVA DO INGLÊS: ESTUDO COMPARATIVO BASEADO EM CORPUS DE FALANTES NATIVOS E APRENDIZES BRASILEIROS DE INGLÊS
}

\author{
THE USE OF DITRANSITIVE CONSTRUCTIONS OF \\ ENGLISH: A COMPARATIVE STUDY BASED ON ENGLISH \\ NATIVE SPEAKER AND BRAZILIAN LEARNER CORPORA
}

Vanessa Cristina Oliveira Wright"

Resumo: Este trabalho investigou o uso da construção bitransitiva por aprendizes brasileiros de inglês e comparou-o com o de falantes nativos do inglês. Para tanto, utilizamos dois corpora de escrita acadêmica. O corpus de aprendizes selecionado foi o BR-ICLE (160.000 palavras), e o corpus de falantes nativos selecionado foi o LOCNESS (320.000 palavras). Para fazer a seleção e análise dos dados utilizamos a ferramenta WordSmith tools. Trabalhamos com o conceito de gramática de construção de Goldberg (1995) e com os conceitos de bi-transitividade de Carter e McCarthy (2006), e de Downing e Locke (2006). Os dados nos mostraram que os aprendizes brasileiros utilizaram a forma bitransitiva, porém com algumas influências do português. O que também diferenciou o uso da construção bitransitiva nos dois corpora foi a frequência de certos tipos de SN do objeto indireto.

Palavras-chave: Gramática de Construção; Bitransitividade; Corpus de aprendiz.

AвSTRACT: This study has investigated the use of ditransitive constructions by Brazilian learners of English, and compared it with native English speaker's constructions. In order to do this analysis, we used two corpora of academic writing. We selected the learner's corpus BR-ICLE (160,000 words), and the native speaker' corpus LOCNESS (320,000 words). The corpus linguistics tool WordSmith was used for selection the data. We worked with Goldberg's construction grammar concept (1995), as well as Carter and McCarthy (2006)'s and Downing and Locke (2006)'s concepts of bi-transitivity. The data showed us that the Brazilian learners did use the ditransitive form V SN SN as the natives, showing some Portuguese influence. The frequency of certain types of SN indirect object was also different between natives and Brazilian learners.

KEYWORDS: Construction Grammar; Ditransitive construction; Learner corpus.

"Doutoranda em Estudos Linguísticos. Universidade Federal de Minas Gerais - UFMG. Email: vcowright@gmail.com 


\section{CONSIDERAÇÕES INICIAIS}

O modelo de gramática de construções proposto por Goldberg (1995) foi um grande avanço nos estudos de linguística cognitiva. Tendo em vista os conceitos propostos pela autora, analisamos as construções bitransitivas produzidas por aprendizes brasileiros de inglês e comparamos com a produção de falantes nativos do inglês. Para isso, utilizamos dois corpora: um de aprendiz (BR-ICLE) com aproximadamente 160 mil palavras, e um de falantes nativos do inglês (LOCNESS), com aproximadamente 320 mil palavras.

Este estudo foi construído para responder à seguinte pergunta: os aprendizes brasileiros utilizam a construção bitransitiva V SN SN com frequência? Ou eles dão preferência para a construção V SN SPrep? Tal pergunta surgiu devido às diferenças entre tais construções bitransitivas do inglês e do português. Tendo em vista uma possível influência da língua materna, imaginamos que os aprendizes dariam preferência à construção com o SPrep, em detrimentos da construção V SN SN.

Para Goldberg (1995) uma construção não é apenas a soma das partes, ela é o pareamento entre léxico e gramática. Dentre algumas construções do inglês que ela propõe, temos a construção bitransitiva. Para ela, essa construção pode ocorrer de 2 formas. A primeira sendo $\mathrm{V}$ (verbo) SN (sintagma nominal) SN (sintagma nominal) e a segunda V (verbo) SN (sintagma nominal) SPrep (Sintagma Preposicionado). Essas duas formas da construção bitransitiva têm algumas diferenças semânticas que serão discutidas na próxima sessão. Também trabalhamos com a definição de bitransitividade de Carter e McCarthy (2006) e de Downing e Locke (2006). Esses dois trabalhos consistem em gramáticas descritivas da língua inglesa baseadas em corpus oral e escrito.

Por se tratar de um estudo baseado na língua em uso de aprendizes, este trabalho pretende ampliar o conhecimento acerca das características da escrita acadêmica de aprendizes brasileiros de inglês. O estudo de corpus de escrita acadêmica de aprendizes brasileiros é relativamente recente (DUTRA; SILERO; 2010), tendo crescido mais significantemente a partir da década de 90. Ao descrevermos a língua alvo utilizada pelos aprendizes, tornamos possível a identificação de características dos aprendizes, além da identificação de erros, permitindo o conhecimento da interlíngua dos aprendizes.

Uma coletânea importante para essa área é a Granger (1998), onde encontramos pesquisas baseadas em corpus de aprendizes, e suas aplicações no ensino e aprendizagem. Geoffrey Leech (1998) afirma no prefácio dessa obra que o estudo de corpus de falantes não nativos é muito novo, e que há, ainda, uma falta grave de conexão com os estudos com as noções das teorias linguísticas mais correntes.

Nessa coletânea destacam-se trabalhos como o de Meunier (1998) que fornece uma crítica sobre várias ferramentas para o trabalho com diferentes corpora, além de discutir os tipos de softwares disponíveis para análises de frequência lexical e estilos de concordância. Destaca-se, também, o estudo de Altenberg e Tapper (1998), onde discutem a super utilização e falta de utilização de 
certas conjunções por aprendizes suécos de inglês. E, também, Gillard e Gadsby (1998), que analisam o uso de corpus de aprendiz para a criação de dicionários para o ensino da língua inglesa.

A pesquisa aqui proposta vem se unir a esses trabalhos mencionados e a outros que contribuem para uma maior compreensão da linguagem utilizada por aprendizes de inglês, e também de aprendizes brasileiros da língua inglesa, proporcionado a chance de reflexão sobre as características dessa linguagem, suas motivações e possibilidades de alteração de padrões indesejados.

Dividimos esse artigo em três sessões. A primeira traz as teorias que norteiam esse trabalho, assim como as definições que utilizamos para analisar os dados. A segunda é uma descrição dos corpora selecionados para a pesquisa e uma descrição da ferramenta utilizada para fazer a seleção dos dados dos corpora, juntamente com os procedimentos metodológicos adotados para limpeza dos dados. Por último, a terceira sessão traz a descrição e análise dos resultados.

\section{REFERENCIAL TEÓRICO}

\section{CoNCEIRO DE CONSTRUÇÃO}

Este estudo adotou o modelo de gramática de construção proposto por Goldberg (1995). Esse conceito de construções já não era algo novo e próprio da teoria da gramática de construções (GOLDBERG, 1995). No estudo de gramática gerativa, o uso de construções já era utilizado, e até mesmo antes, nos estudos de gramática tradicional. $\mathrm{Na}$ gramática de construções de Goldberg, uma construção é definida como uma unidade básica da língua e essa unidade é encontrada em todos os níveis da língua, desde o nível morfológico até o nível sintático e semântico. O que é novo nessa gramática de construções é o papel do léxico e da gramática.

Para Goldberg (1995),

In Construction Grammar, no strict division is assumed between the lexicon and syntax. Lexical constructions and syntactic constructions differ in internal complexity, and also in the extent to which phonological form is specified, but both lexical and syntactic constructions are essentially the same type of declaratively represented data structure: both pair form with meaning. (GOLDBERG, 1995, p.7)1

A construção, que é uma unidade linguística (GOLDBERG, 1995), é expressa através de padrões frasais. Esses padrões podem ser considerados construções quando seu significado não é previsível a partir de suas partes, ou das propriedades de suas partes. Outro fator que deve ser levado em conta é se outras construções também não podem prever o sentido dessas estruturas.

Segundo a autora, há algumas vantagens em descrever a língua através da gramática das construções. A primeira delas é que sentidos implausíveis para verbos são evitados. Isso acontece nas teorias em que,

\footnotetext{
${ }^{1}$ Tradução nossa: na gramática de construção, nenhuma divisão estrita é assumida entre o léxico e a sintaxe. Construções lexicais e construções sintáticas diferem-se em sua complexidade interna, e também na extensão na qual a forma fonológica é especificada, mas ambas construções sintáticas e lexicais são essencialmente o mesmo tipo de estrutura representativa de dados declarativos: ambos fazem par com o significado.
} 
sempre que um verbo é usado com um sentido desconhecido, esse sentido novo é adicionado à diátese do verbo. Um exemplo que a autora fornece é o famoso She sneezed the napkin off the table, em que o verbo tradicionalmente intransitivo sneeze ocorre em uma construção transitiva. Segundo a teoria das construções, ao invés de incluir mais esse significado de sneeze na diátese do verbo, o verbo aparece em uma construção (um padrão frasal). No caso do exemplo acima, a construção do inglês seria $\mathrm{X}$ causa movimento de Y através de uma ação (no caso sneeze). A segunda vantagem seria a circularidade que é evitada. A circularidade ocorre em teorias que afirmam que a sintaxe é uma projeção de requisitos lexicais. Com isso, elas utilizam a sintaxe para explicar fenômenos lexicais, mas usam, também, fenômenos sintáticos para explicar o léxico. Com a gramática de construções esse fenômeno seria evitado. A terceira vantagem é em relação à parcimônia semântica, já que se acredita que sentidos diferentes de um verbo não existem. Por último, a quarta vantagem da gramática de construções é que a composicionalidade é preservada, pois uma construção só vai ser considerada uma construção se algo sobre sua forma, significado, ou uso não for previsível através de outros aspectos da gramática, incluindo outras construções já existentes.

Por esses motivos, Goldberg (1995) afirma que utilizar os conceitos de gramática de construção seria uma solução para alguns problemas encontrados por outras teorias que acabam sendo explicadas como exceção. Seria, também, uma forma de explicar como esses fenômenos ocorrem, mostrando suas regularidades. Vale lembrar que, embora as construções tenham significado independente, elas não impõem seus significados ao verbo, visto que o verbo também predica. Dessa forma, segundo Goldberg (1995), as análises devem ser feitas de ambas as formas (top-down e bottom-up), levando em consideração tanto o verbo quanto a construção.

\section{A CONSTRUÇÃO BITRANSITIVA}

Goldberg (1995) fala de construções de estrutura argumental, que são as orações compostas por verbos e seus argumentos. Para a autora, a construção bitransitiva pode ocorrer de duas formas (GOLDBERG, 1995):

1. V SN SN, em que o primeiro SN é o recebedor (objeto indireto) e o segundo SN é o objeto direto.

2. V SN SPrep, em que o SPrep é o recebedor (objeto preposicionado) e o segundo SN é o objeto direto.

A construção bi-transitiva, ou "construção de duplo objeto" (FERRARI, 2011, p.135) consiste em uma construção gramatical em que o verbo terá dois argumentos, com a seguinte estrutura: $\mathrm{X}$ causa $\mathrm{Y}$ a receber $\mathrm{Z}$, exemplificado a seguir pelos exemplos (a) e (b):

\section{(a) Mary showed her friend a picture. \\ (b) Mary showed a picture to her friend.}

No exemplo (a), her é a beneficiária, já que ela recebe o presente, e em (b), to her friend é a beneficiária. Por isso, os papéis temáticos de $Y$ na construção bi-transitiva serão de recebedor ou de beneficiário. Segundo 
Goldberg (1995), o uso da forma 1, ou da forma 2 implica diferentes interpretações semânticas. De acordo com a definição da autora, a oração (a) implica que a amiga viu a foto, já na oração (b) não tem essa implicação (Mary showed a picture to her friend, but she was almost asleep).

Carter e McCarthy (2006) descrevem a complementação bitransitiva como as combinações de 2 argumentos, o objeto direto e o indireto, ou o objeto direto e objeto preposicionado, e acrescenta que o objeto indireto sempre deve vir acompanhado de um objeto direto. Além disso, os autores afirmam que o objeto indireto geralmente ocorre com um objeto animado. Porém, para eles, o uso do objeto indireto ou preposicionado é uma questão de enfoque, já que

The choice between using an indirect + direct structure or an object + prepositional phrase (oblique complement) depends on what the speaker wishes to focus on in the message. As end position in English is generally associated with gender focus, either the direct (in green) of the verb can be put into focus or the recipient of the direct object (underlined):

George handed William the bottle. (end-focus on the bottle)

George handed the bottle to William. (end-focus on William) (CARTER; McCARTHY, 2006, p.521) ${ }^{2}$

\footnotetext{
${ }^{2}$ Tradução nossa: a escolha entre usar uma estrutura com objeto indireto + direto ou um objeto + frase preposicionada (complemento oblíquo) depende do quê o falante deseja focar na mensagem. Como a posição final do inglês é geralmente associada com maior foco, ou o objeto do verbo (em negrito) pode ser colocado em foco ou o recipiente do objeto direto (sublinhado):
}

Downing e Locke (2006) partilham dessa distinção e complementam que os verbos de transferência são os verbos que tipicamente ocorrem com dois objetos, e cita como exemplos os verbos give, hand, lend, offer, owe, pass, promise, read, send, show, teach, dentre outros. Ainda segundo os autores, a estrutura bitransitiva também ocorre com outros tipos de verbos, como os verbos de intenção de transferência, prestação de serviços, dentre outros.

Acreditamos que as distinções entre o tipo de bitransitivo dado por Goldberg (1995) e por Carter e McCarthy (2006) não são incompatíveis, podendo haver uma ou outra interpretação, dependendo do contexto, assim como da proficiência do falante.

Na próxima sessão, descreveremos como selecionamos os verbos mais frequentes que prototipicamente ocorrem nesse tipo de construção.

\section{Metodologia}

Os estudos baseados em corpus têm ganhado destaque atualmente, juntamente com os estudos baseados em corpus de aprendizes. De acordo com Bonelli (2010), esse tipo de corpus pode ser comparado com corpus de falantes nativos e a língua dos aprendizes pode ser explorada de uma forma muito mais profunda. Um dos principais projetos na Europa de corpus de aprendizes é o projeto ICLE da Universidade de Louvian, na Bélgica, corpus do qual o BR-ICLE (copus de

George handed William the bottle. (foco final em the bottle)

George handed the bottle to William. (foco final em William) (foco final em the bottle) 
estudo escolhido para esse trabalho) faz parte e para cuja compilação a PUC-SP colabora.

Para compararmos as construções produzidas, escolhemos um corpus de referência de falantes nativos do inglês (LOCNESS), e um corpus de estudo de aprendizes brasileiros do inglês (BR-ICLE).

O BR-ICLE3 é um corpus em construção, composto de ensaios de aprendizes brasileiros da língua inglesa que faz parte do projeto ICLE. O Projeto ICLE consiste em vários subcorpora compostos por textos de escrita acadêmica em inglês, de falantes aprendizes dessa língua, cuja língua materna seja outra. Esse projeto possui vários parceiros espalhados pelo mundo, ligados a universidades em vários países. Há um total de 24 subcorpora, representantes de línguas maternas diferentes. O BR-ICLE é o subcorpus que representa o português do Brasil, possuindo atualmente aproximadamente 160 mil palavras.

O LOCNESS4 é um corpus de ensaios argumentativos de estudantes universitários britânicos e americanos. Ele é composto por 324.304 palavras, sendo 60.209 palavras de estudantes britânicos, 95.695 palavras de universitários britânicos e 168.400 palavras de universitários americanos.

Para selecionar os dados dos corpora escolhidos, utilizamos a ferramenta Wordsmith Tools. Ela é uma ferramenta que permite analisar corpora a partir de funções como concordâncias, listas de frequências, listas de palavras individuais e múltiplas, colocações

${ }^{3}$ Informações retiradas do site http://www.uclouvain.be/ en-317607.html, último acesso em 06/06/2015

${ }^{4}$ Informações retiradas do site http://www.uclouvain.be/ en-cecl-locness.html, último acesso em 06/06/2015 e outras. Esse software pode ser adquirido via download no endereço http://www.lexically. net/wordsmith/.

O Wordsmith Tools (SARDINHA, 2006) possui 3 (três) ferramentas básicas: wordList, o keywords e o concord. Selecionamos os verbos bi-transitivos mais frequentes com base na lista de palavras gerada com o WordList. Essa ferramenta cria uma lista com todas as palavras do corpus e a organiza em ordem decrescente de frequência. Primeiro identificamos os 2 verbos bitransitivos mais frequentes no corpus de referência. Em seguida, pesquisamos esses verbos no corpus com a ferramenta Concord tanto no LOCNESS como no BR-ICLE para obter as orações em que os verbos ocorreram. Todas as formas dos verbos foram selecionadas, e os dados que não fariam parte dessa pesquisa foram excluídos, como phrasal verbs que continham os verbos selecionados e apareceram nas linhas de concordância. Também não entraram na análise dos dados os verbos na voz passiva.

Após essa etapa, prosseguimos com a análise dos dados que serão descritos na seção 3 .

\section{DESCRIÇÃO E ANÁLISE DOS DADOS}

Os dois verbos mais frequentes que geralmente ocorrem em uma construção bitransitiva no corpus de falantes nativos foram os verbos show e give.

Tabela 1: Frequência do verbo show nos corpora

\begin{tabular}{l|c|c|c}
\hline Verbo & Nativo & Aprendiz & $\begin{array}{c}\text { Aprendiz } \\
\text { (Normalizada) }\end{array}$ \\
\hline Show & 142 & 122 & 244 \\
\hline
\end{tabular}


Os valores da frequência dos verbos apresentados nas tabelas referem-se às ocorrências em uma oração, ou seja, cada ocorrência é uma oração em que o verbo show ou give eram os predicadores da oração. A tabela I mostra as ocorrências do verbo show no corpus de falantes nativos e no corpus de aprendizes.

Os valores do corpus de aprendizes foram normalizados para que pudéssemos comparar com os resultados encontrados no corpus de falantes nativos. Como o LOCNESS é 2,01 vezes maior que o $B R-I C L E$, os resultados do BR-ICLE foram normalizados multiplicando por 2,01.

A tabela 2 mostra a frequência das construções com o verbo show. Apesar de os aprendizes utilizarem mais esse verbo, a distribuição da frequência dos tipos de construção foi semelhante entre os dois grupos. Ambos produziram mais construções transitivas com o verbo show.

Tabela 2: Frequência das construções com o verbo show

\begin{tabular}{l|c|c|c|c|c|c|c|c}
\hline & V+SN+SN & $\%$ & V+SN+Sprep & $\%$ & V+SN & $\%$ & V+Sprep+SN & $\%$ \\
\hline Nativo & 28 & 20 & 0 & 0 & 114 & 80 & 0 & 0 \\
\hline $\begin{array}{l}\text { Aprendiz } \\
\text { (Normalizado) }\end{array}$ & 34 & 14 & 0 & 0 & 202 & 83 & 8 & 3 \\
\hline
\end{tabular}

O que se mostrou diferente entre os nativos e os aprendizes foi o uso de uma forma da construção bitransitiva pelos aprendizes. Os aprendizes utilizaram uma forma $\mathrm{V}+$ Sprep+SN como sendo uma construção bitransitiva.

(c) They need to be realistic and show to the students what they really need (BR-ICLE)

(d) This university should show to these students things that are going to help them (BR-ICLE).

(e) They showed to everyone that they can do (BR-ICLE).

Essas ocorrências foram produzidas por informantes diferentes, o que pode indicar que eles utilizaram uma forma comum da construção bitransitiva do português na produção do inglês, conforme tradução do exemplo (e) "Eles mostraram a todos que eles eram capazes".

Outro fato interessante foi que a forma $\mathrm{V}+\mathrm{SN}+\mathrm{SPrep}$ da construção bitransitiva não foi utilizada com o verbo show em nenhum dos dois corpora e que o verbo show foi mais utilizado com a construção transitiva, conforme exemplos (f) e (g):

(f) Some television shows show violence because it appeals to adult viewers. (LOCNESS).

(g) This essay intends to show some aspects the learning (BR-ICLE).

O que mostra que o verbo pode ter influência na seleção do tipo de construção que ele irá ocorrer (diátese). 
Tabela 3: Frequência da construção bitransitiva com show

\begin{tabular}{l|c|c|c|c}
\hline & $\begin{array}{l}\text { V+Pronome } \\
\text { Pessoal+SN }\end{array}$ & $\%$ & V+Nome+SN & $\%$ \\
\hline Nativo & 19 & 68 & 9 & 32 \\
\hline $\begin{array}{l}\text { Aprendiz } \\
\text { (normalizado) }\end{array}$ & 30 & 88 & 4 & 12 \\
\hline
\end{tabular}

A diferença mais significativa, na produção dos falantes nativos e dos aprendizes brasileiros com a construção bitransitiva $\mathrm{V}$ SN SN foi nos tipos de SN utilizados no primeiro SN (objeto indireto) da construção, conforme exemplos:

(h) Caligula not only wants to show his people the one truth (LOCNESS).

(i) They show us a false world (BR-ICLE).

Embora ambos os corpora tenha apresentado preferência pelos pronomes, os aprendizes brasileiros utilizaram SN composto por pronomes pessoais $20 \%$ a mais que os nativos, conforme tabela 3. Esses dados, porém, não são suficientes para criar uma hipótese sobre o porquê dessa preferência.

O verbo give apresentou ocorrências um pouco diferentes do verbo show. A tabela 4 mostra que a frequência do uso desse verbo entre os falantes nativos e aprendizes foi muito semelhante.

Tabela 4: Frequência do verbo give nos corpora

\begin{tabular}{c|c|c|c}
\hline Verbo & Nativo & Aprendiz & $\begin{array}{c}\text { Aprendiz } \\
\text { (Normalizada) }\end{array}$ \\
\hline Give & 233 & 113 & 226 \\
\hline
\end{tabular}

Além disso, a forma $\mathrm{V}+\mathrm{SN}+\mathrm{SPrep} \mathrm{da}$ construção bitransitiva foi utilizada com o verbo give, diferentemente do verbo show. A tabela 5 mostra que o verbo give é mais usado na construção bitransitiva, porém ele ocorre com uma frequência significativa na forma transitiva também.

Tabela 5: Frequência das construções com o verbo give

\begin{tabular}{l|c|c|c|c|c|c|c|c}
\hline & V+SN+SN & $\%$ & V+SN+Sprep & $\%$ & V+SN & $\%$ & V & $\%$ \\
\hline Nativo & 130 & 56 & 57 & 24 & 46 & 20 & 0 & 0 \\
\hline Aprendiz (Normalizada) & 120 & 53 & 62 & 27 & 46 & 20 & 4 & 2 \\
\hline
\end{tabular}

j) Foxes give chickens a cruel death (LOCNESS).

k) Many times these women can not give a good life to the baby (BR-ICLE).

l) I have to give a class for the first time. (BR-ICLE).

m) Owing the best gift for you to give (BR-ICLE).
Embora os aprendizes tenham produzido algumas orações com verbo give na forma intransitiva, isso ocorreu em uma estrutura em que o objeto foi exposto antes da oração não finita. 
Tabela 6: Frequência da construção bitransitiva com give

\begin{tabular}{l|c|c|c|c}
\hline & V+Pronome+SN & \% & V+Nome+SN & $\%$ \\
\hline Nativo & 73 & 56 & 57 & 44 \\
\hline Aprendiz (Normalizada) & 84 & 70 & 36 & 30 \\
\hline
\end{tabular}

Ao analisarmos as ocorrências do verbo give na construção bitransitiva com objeto indireto, vemos o mesmo ocorrido com o verbo show. Os aprendizes apresentaram uma maior preferência pelo pronome no objeto indireto. Com esse verbo, porém, os nativos apresentaram apenas uma leve preferência pelo pronome, conforme exemplos (n) e (o):

n) It is better to give forteen people a fortune (LOCNESS).

o) They give us reasons for living (BR-ICLE).

Esses dados mostram que, diferentemente do que prevíamos, os aprendizes utilizam sim a construção bitransitiva V SN SN com frequência semelhante à dos nativos. $\mathrm{E}$ que, apesar de a construção ter mais de uma possibilidade de forma, o verbo pode influenciar em que forma ou em que construção ele vai ocorrer com mais frequência.

\section{CONSIDERAÇÕES FINAIS}

Esse trabalho analisou as construções bitransitivas em um corpus de aprendizes brasileiros do inglês e comparou-as com as construções bitransitivas produzidas por falantes nativos do inglês sob a ótica da gramática de construções de Goldberg (1995). Nossa hipótese era que os aprendizes brasileiros utilizariam a construção bitransitiva V SN SN com uma frequência muito baixa, dando preferência para a construção bitransitiva V SN SPrep. Para tanto, selecionamos os 2 verbos mais frequentes do corpus de falantes nativos que pudessem ocorrer nessa construção, e analisamos as orações produzidas com esses dois verbos em ambos os corpora (LOCNESS e BR-ICLE). Nossa hipótese não foi confirmada com os resultados, já que os aprendizes utilizaram a construção com grande frequência.

Os resultados também mostraram que a frequência de verbos em cada construção varia de acordo com o verbo. Os 2 verbos mais frequentes (show e give) apresentaram frequências bem distintas em cada construção (bitransitiva ou transitiva). Essa diferença foi observada tanto no corpus de aprendizes (BR-ICLE) quanto no corpus de falantes nativos (LOCNESS).

Outro ponto observado foi em relação à influência da língua materna na escrita de alguns aprendizes brasileiros. Embora a construção bitransitiva apenas aceite V SN SN ou V SN SPrep, encontramos nos dados de aprendizes algumas ocorrências da estrutura V SPrep SN, em que o SPrep era o objeto indireto, e o SN o objeto direto. Essa estrutura não foi encontrada no corpus de falantes nativos, e acreditamos que tenha sido por influência do português, que aceita essa inversão de objetos.

A última questão analisada foi a diferença quantitativa com a construção 
bitransitiva V SN SN entre os nativos e aprendizes. Embora ambos os grupos tenham demonstrado uma preferência pelo primeiro SN realizado por um pronome, os aprendizes produziram muito mais pronomes que os nativos. Não encontramos, porém, algo que explicasse essa diferença, com base nos dados obtidos.

Esse trabalho apresentou algumas limitações. Primeiramente, o número limitado de verbos na construção analisada. Um estudo mais aprofundado, com mais verbos, incluindo aqueles que não ocorrem na forma bitransitiva no português seria necessário para fazer afirmações mais precisas acerca do aprendizado da construção bitransitiva do inglês por aprendizes brasileiros. Outro aspecto não analisado nesse trabalho foi o tipo de nome que ocorreu no primeiro $\mathrm{SN}$. Como o objeto indireto geralmente ocorre com um objeto animado (Carter; McCarthy, 2006), seria interessante analisar, também, se há diferenças no tipo de nome utilizado entre os aprendizes e nativos. Apesar dessas limitações, acreditamos que este trabalho seja uma contribuição para a descrição da língua de aprendizes brasileiros dentro dos estudos da gramática de construções.

\section{REFERÊNCIAS}

ALTENBERG, B.; TAPPER, M. The use of adverbial connectors in advanced Swedish learners' written English. In: GRANGER, S. (Ed.). Learner English on Computer. London \& New York: Addison Wesley Longman, 1998. p. 80-93.

BONELLI, E. T.; Theoretical overview of the evolution of corpus linguistics. In: The
Routledge handbook of Corpus Linguistics, Routledge, 2010.

CARTER, R.; McCARTHY, M.. Cambridge Grammar of English: A Comprehensive Guide - Spoken and Written English Grammar and Usage. Cambridge University Press, 2007 DOWNING, A.; LOCKE, P. English Grammar: A university Course. Second Edition, Taylor \& Francis e-Library. 2006.

DUTRA, D. P.; SILERO, R. P. Descobertas linguísticas para pesquisadores e aprendizes: a linguística de Corpus e o ensino de gramática. In Revista Brasileira de Linguística Aplicada, Vol. 10, no. 4, Belo Horizonte, 2010.

FERRARI, L. Introdução à Linguística Cognitiva. São Paulo: Contexto, 2011. GILLARD, P; GADSBY, A. Using a learners' corpus in compiling ELT dictionaries. In: GRANGER, S. (Ed.). Learner English on Computer. London \& New York: Addison Wesley Longman, 1998. p.159-171.

GOLDBERG, A. E. Constructions: a construction grammar approach to argument structure. Chicago: The University of Chicago Press, 1995.

MEUNIER, F. Computer tools for the analysis of learner corpora. In: GRANGER, S. (Ed.). Learner English on Computer. London \& New York: Addison Wesley Longman, 1998. p. 19-38. SARDINHA, T. B. Pesquisa em Linguística de Corpus com Wordsmith Tools. 2006.

Recebido para publicação em 12 out. 2015. Aceito para publicação em 18 ago. 2016. 